\title{
O efeito em curto prazo do bypass gástrico sobre pacientes obesos diabéticos
}

\section{Short-term effect of gastric bypass in obese diabetic patients}

\author{
Aluisio Stoll, TCBC-SC1; Jean Carl Silva²; luiz Carlos Von Bahten, TCBC-PR³; Giovana Gugelmin4; André Berton Vedan 5 ; \\ Bárbara Vicente De Souza ${ }^{6}$
}

\section{R E S U M O}

\begin{abstract}
Objetivo: estudar o efeito do bypass gástrico sobre a glicemia e o uso de medicação antidiabética em pacientes obesos portadores de diabetes. Métodos: estudo de coorte retrospectivo com 44 pacientes obesos portadores de DM2, provenientes de 469 pacientes submetidos ao bypass gástrico no período de dezembro de 2001 a março de 2009. Os desfechos primários avaliados foram: glicemia em jejum e a necessidade de medicação antidiabética. Resultados: a população foi composta de dez (22,7\%) homens e $34(77,3 \%)$ mulheres, com média de idade de $45,3(+8,23)$ anos e índice de massa corporal de 40,9 (+5,03) kg/m $\square$. 0 tempo médio de evolução do DM2 foi 63,6 (+60,9) meses. Dos 40 pacientes que utilizavam medicação para controle do DM2, 20 (50\%) tiveram sua medicação suspensa na alta hospitalar e 13 (32,5\%) até nove meses depois. Em uma paciente não foi possível avaliar o uso de medicação, sendo essa a única exclusão. A insulina foi suspensa nos dez (100\%) pacientes que a utilizavam, sendo seis (60\%) na alta hospitalar. Houve redução $(P<0,05)$ da glicemia em jejum, em todo o período estudado, em comparação com o valor pré-operatório, e foram atingidos valores inferiores a $100 \mathrm{mg} / \mathrm{dl}$ no período de sete a nove meses. Conclusão: Pacientes obesos portadores de DM2, submetidos ao bypass gástrico, apresentaram melhora do controle glicêmico e redução do uso de hipoglicemiantes em curto prazo.
\end{abstract}

Descritores: Pacientes. Obesidade. Diabetes mellitus tipo 2. Glicemia. Derivação Gástrica.

\section{INTRODUÇÃO}

O diabetes mellitus (DM) é um distúrbio crônico, caracterizado pelo comprometimento do metabolismo da glicose, que resulta em hiperglicemia com suas consequentes complicações'.

O DM tipo 2 (DM2) é, sem dúvida, a apresentação mais comum da doença, encontrado em mais de $90 \%$ da população diabética. Tipicamente surge depois dos 40 anos de idade e principalmente associado à obesidade ${ }^{2}$.

A prevalência da obesidade e do DM2 aumentou exponencialmente, constituindo um dos maiores problemas atuais de saúde pública ${ }^{3}$. O tratamento cirúrgico da obesidade mórbida acarreta além da perda de peso, o controle das comorbidades e principalmente, do $\mathrm{DM} 2^{4}$.

Estudos recentes têm interrogado o mecanismo pelo qual pacientes obesos mórbidos controlam o DM2 após cirurgias bariátricas. A maioria desses pacientes recebe alta hospitalar após a operação, sem a necessidade de medicamentos para o DM2, quando ainda não perdeu peso.
Na realidade, a perda de peso é parcialmente responsável pela reversão do DM2. A derivação intestinal que é realizada nessas operações provoca um efeito imediato de melhora e um aumento da produção de insulina, devido à produção acentuada de (GLP-1) glucagon-like peptide-1 e à ação melhorada do (GIP) gastric inhibitory polypeptide, ou glucose-dependent insulinotropic polypeptide, que contribuem para a melhora dos níveis glicêmicos e, consequentemente, do $\mathrm{DM} 2^{5-8}$.

O impacto da cirurgia bariátrica sobre a melhoria do DM2 talvez possa assemelhar-se à descoberta da insulina, e, certamente, estamos vivendo um novo momento histórico da medicina. O objetivo deste estudo foi avaliar os efeitos do bypass gástrico em Y de Roux sobre a glicemia e o uso de hipoglicemiantes em pacientes obesos portadores de DM2.

\section{MÉTODOS}

Foi realizado um estudo de coorte retrospectivo com pacientes obesos portadores de DM2 submetidos ao

Trabalho realizado no Instituto de Cirurgia Digestiva e Obesidade de Joinville (ICDO).

1. Cirurgião Geral do Instituto de Cirurgia Digestiva e Obesidade de Joinville(ICDO); 2. Professor do Departamento de Medicina da Universidade da Região de Joinville; 3. Coordenador do Programa de Mestrado em Cirurgia PUCPR; 4. Residente de Cirurgia Geral do Hospital Municipal São José, Joinville SC; 5 . Residente de Cirurgia Geral da Santa Casa de Misericórdia, Ponta Grossa PR; 6. Endocrinologista do Instituto de Endocrinologia de Joinville (IEJE). 
bypass gástrico em $Y$ de Roux, no período de 11 de dezembro de 2001 a 30 de março de 2009, em que foram avaliados os desfechos primários: a glicemia plasmática em jejum e o uso dos antidiabéticos. O estudo seguiu as recomendações da Resolução nº 196/96 do Conselho Nacional de Saúde, foi aprovado e registrado sob 0 n 080025 do Comitê de Ética em Pesquisa do Hospital Regional Hans Dieter Schmidt (Joinville, SC).

Os critérios de inclusão foram: pacientes portadores de DM2 e obesidade grau III (IMC $\left.>40 \mathrm{~kg} / \mathrm{m}^{2}\right)$, ou obesidade grau II (IMC 35 a 39,9kg/m²), submetidos ao bypass gástrico com alça biliopancreática de $50 \mathrm{~cm}$ e alça alimentar em Y de Roux de $100 \mathrm{~cm}$.

O diagnóstico de DM seguiu os critérios propostos pela Associação Americana de Diabetes (ADA) ${ }^{1}$.

Todos os dados foram coletados nos prontuários médicos: idade, sexo, tempo de diagnóstico de diabetes, glicemia plasmática em jejum, necessidade de medicações antidiabéticas, peso, IMC (peso/altura²) e a percentagem da perda do excesso de peso (PEP). Foram agrupados em intervalos regulares de avaliação: um mês, dois a três meses, quatro a seis meses, sete a nove meses e dez a 12 meses após a operação.

Os dados coletados foram analisados por estatística descritiva, através de médias e desvio-padrão, e pelo teste $t$ com duas amostras em par para médias com nível de significância de $5 \%$ e teste de correlação.

\section{RESULTADOS}

Foram realizados, sem mortalidade, 469 bypass gástricos em Y de Roux. Desses, 44 pacientes tinham diagnóstico de diabetes tipo 20. A população de estudo $(n=44)$ foi composta de dez (22,7\%) homens e 34 (77,3\%) muIheres, com média de idade 45,3 $(+8,23)$ anos e IMC de $40,9(+5,03) \mathrm{kg} / \mathrm{m}^{2}$. O tempo médio de evolução da DM2 foi $63,6(+60,9)$ meses.

Quanto ao uso de medicação para controle do diabetes, foram analisados 43 pacientes, dos quais 40 (93\%) usavam medicação e três (7\%) controlavam apenas com dieta e atividade física. O controle apenas com medicação oral era feito por 30 indivíduos (75\%), com insulina associada à medicação oral por nove $(22,5 \%)$ e, apenas com insulina, um (2,5\%). Em uma paciente, não foi possível avaliar o uso de medicação devido à falta destes dados no prontuário, sendo essa a única exclusão.

A utilização de insulina foi suspensa nos dez pacientes que a utilizavam: seis $(60 \%)$ na alta hospitalar, dois após um mês, e dois após um período de dois meses. Dos 40 pacientes que utilizavam medicação para controle do DM2, 20 (50\%) tiveram sua medicação suspensa na alta hospitalar e mais $13(32,5 \%)$ até nove meses depois. Assim, 33 (82,5\%) indivíduos deixaram de utilizar medicação para controle do DM2, e sete necessitaram continuar (Figura 1).

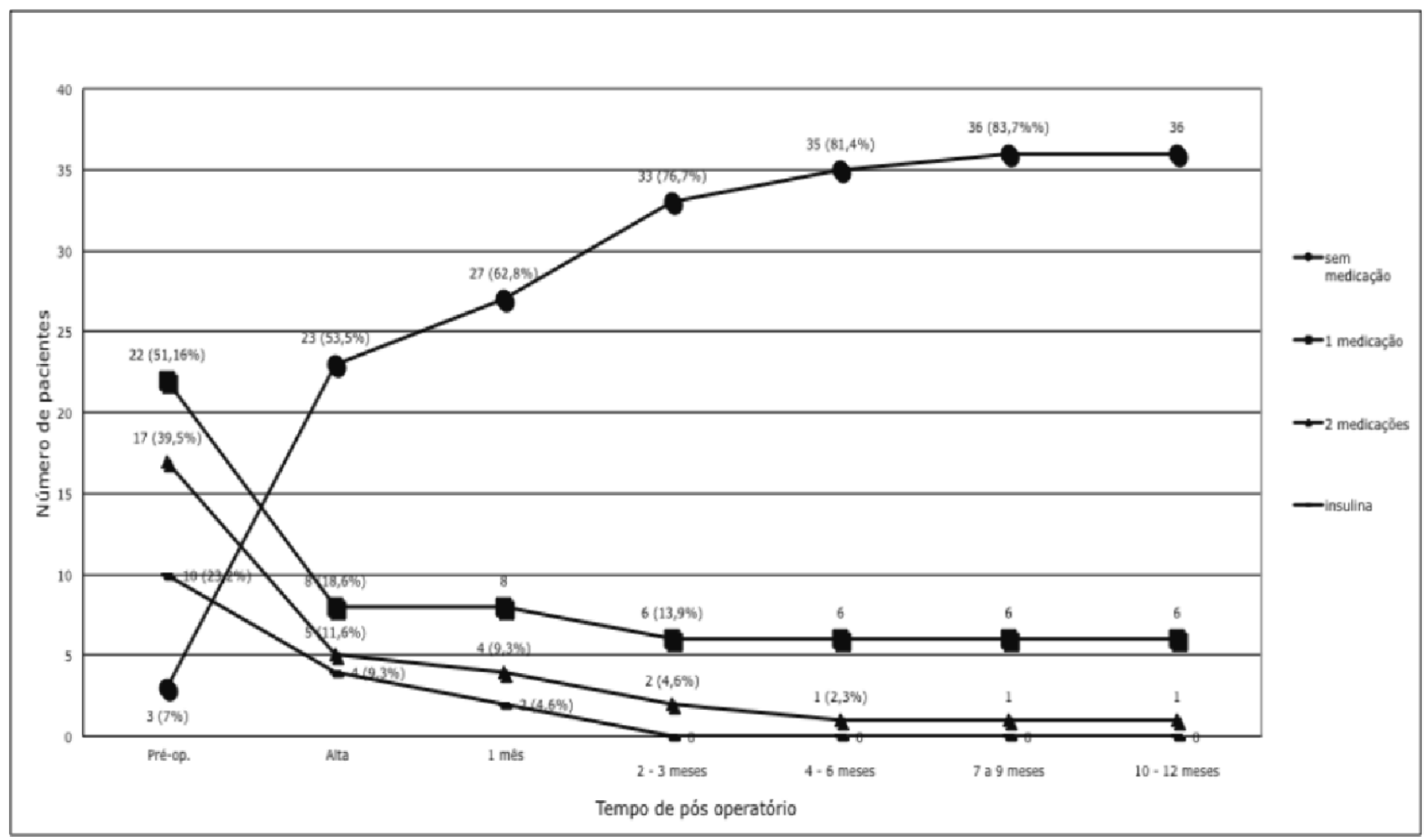

Figura 1 - Utilização de medicação para controle do diabetes nos pacientes submetidos ao bypass gástrico. Pré-op = pré-operatório. 
Em comparação com o valor pré-operatório, houve redução $(P<0,05)$ da glicemia de jejum desde a primeira avaliação, com um mês de pós-operatório. O resultado manteve-se assim em todos os momentos do período estudado. No período de sete a nove meses, as médias das glicemias de jejum apresentaram valores inferiores a $100 \mathrm{mg} / \mathrm{dl}$. Ao final de 12 meses, houve redução $(p<0,01)$ da média da glicemia de jejum do pré-operatório de 151,85 + 45,96 para 89,68 + 18,13 (Figura 2).

Quanto aos desfechos secundários, na avaliação do peso médio houve uma diminuição $(p<0,01)$, em comparação com o pré-operatório (107,33 + 17,32kg), em todos os momentos avaliados até o final do período $(74,40+14,40 \mathrm{~kg})$. Houve redução do IMC médio de $40,90+5,03 \mathrm{~kg} / \mathrm{m}^{2}$ para $29,01+3,65 \mathrm{~kg} / 2(\mathrm{p}<0,01)$ ao final de 12 meses. Neste período, a percentagem de perda do excesso de peso (PEP) foi $66,95 \%+17,47 \%$. Na análise das curvas, verificou-se um coeficiente positivo na correlação entre a glicemia de jejum e os seguintes fatores: peso $(+0,98)$, IMC $(+0,98)$, e uso da medicação $(+0,94)$. A correlação foi negativa entre glicemia de jejum e PEP (Figura 3).

\section{DISCUSSÃO}

O DM2 é uma doença associada à obesidade mórbida em aproximadamente $20 \%$ dos pacientes ${ }^{4}$. Neste estudo, 9,4\% dos obesos submetidos ao bypass gástrico tinham diabetes, aproximadamente a metade do referido na literatura. Trata-se de um estudo retrospectivo onde foram analisados pacientes operados nos últimos dez anos, cuja indicação principal foi a obesidade mórbida (IMC > 40) e foram incluídas operações realizadas no início da década passada, quando a comunidade médica ainda não havia despertado o interesse pelo efeito da cirurgia bariátrica sobre o diabetes. Alguns exames, como a hemoglobina glicosilada, a insulina e o peptídeo $C$, não faziam parte da rotina pré-operatória, motivo pelo qual não foram avaliados. Pories et al. ${ }^{9}$ publicaram sobre este tema em 1995. Entretanto, foi a partir do trabalho publicado em 2004, por Rubino $^{10}$, que os cirurgiões passaram efetivamente a estudar os efeitos da cirurgia bariátrica sobre o DM2.

No presente estudo, o tempo médio de evolução do DM2 foi 63,6 $(+60,9)$ meses. Nessa série, o menor tempo de história do DM2 contribuiu com os altos índices de melhora do controle glicêmico.

Pacientes com diabetes de curta duração parecem ter resolução mais completa ou sustentada da doença. Um dos fatores responsáveis pela falha no tratamento cirúrgico do obeso mórbido diabético é o tempo de história da doença maior do que dez anos (120 meses) $)^{11,12}$. O bypass gástrico é efetivo na perda de peso, porém sua ação benéfica sobre o DM2 diminui com o maior tempo da doença, devido a menor massa residual de células beta. Esses resultados constituem um bom argumento para propor o tra-

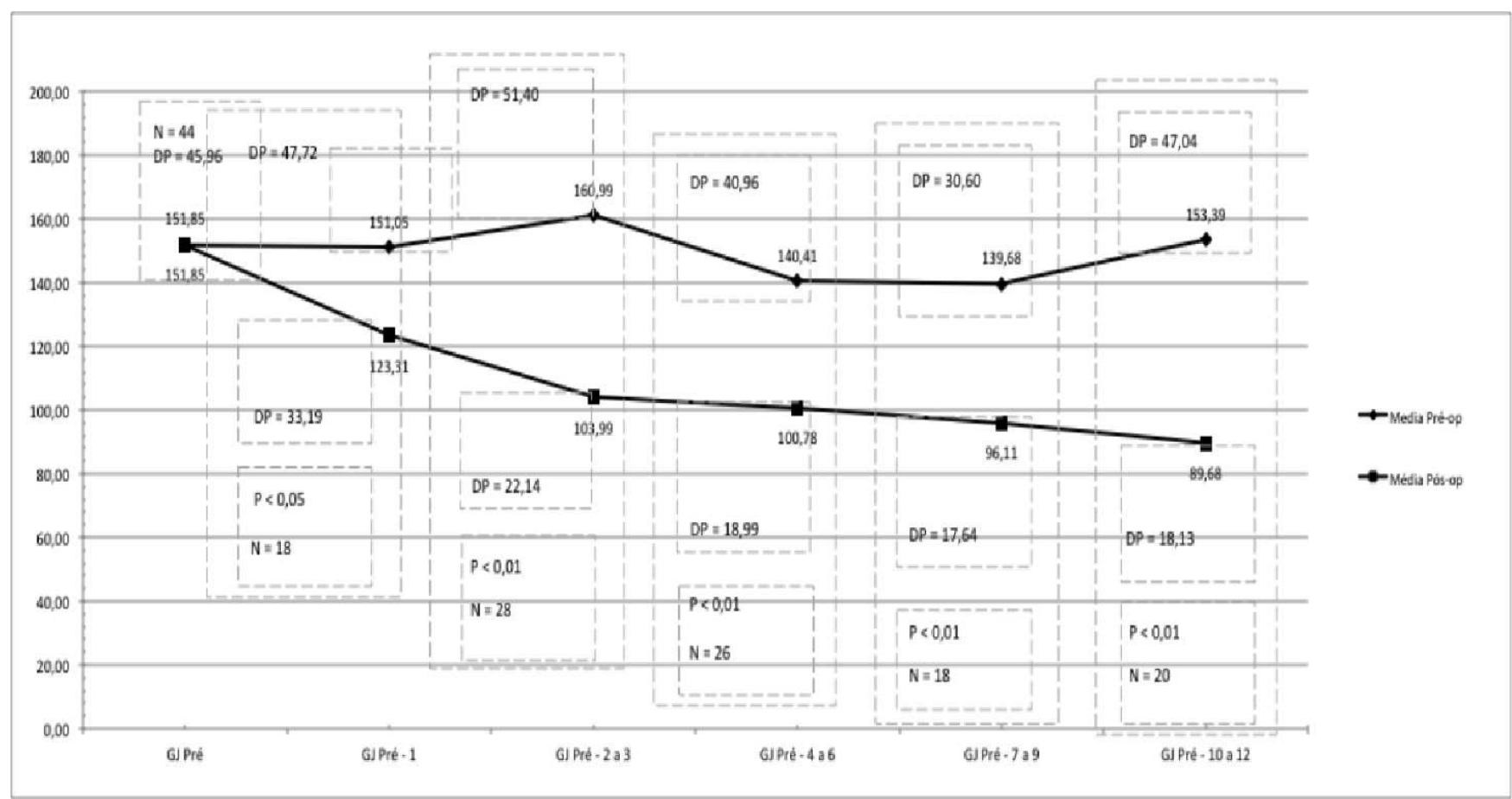

Figura 2 - Comparação da glicemia de jejum do período estudado com seu valor pré-operatório

GJ Pré = Glicemia de jejum pré-operatória; GJ Pré-1= GJ pré X 1 mês de pós-operatório; GJ Pré-2 a 3= GJ pré X 2 a 3 meses de pós-operatório, GJ Pré-4 a 6= GJ pré X 4 a 6 meses de pós-operatório; GJ Pré-7 a 9= GJ pré X 7 a 9 meses de pós-operatório; GJ Pré-10 a $12=$ GJ pré X 10 a 12 meses de pós-operatório. 


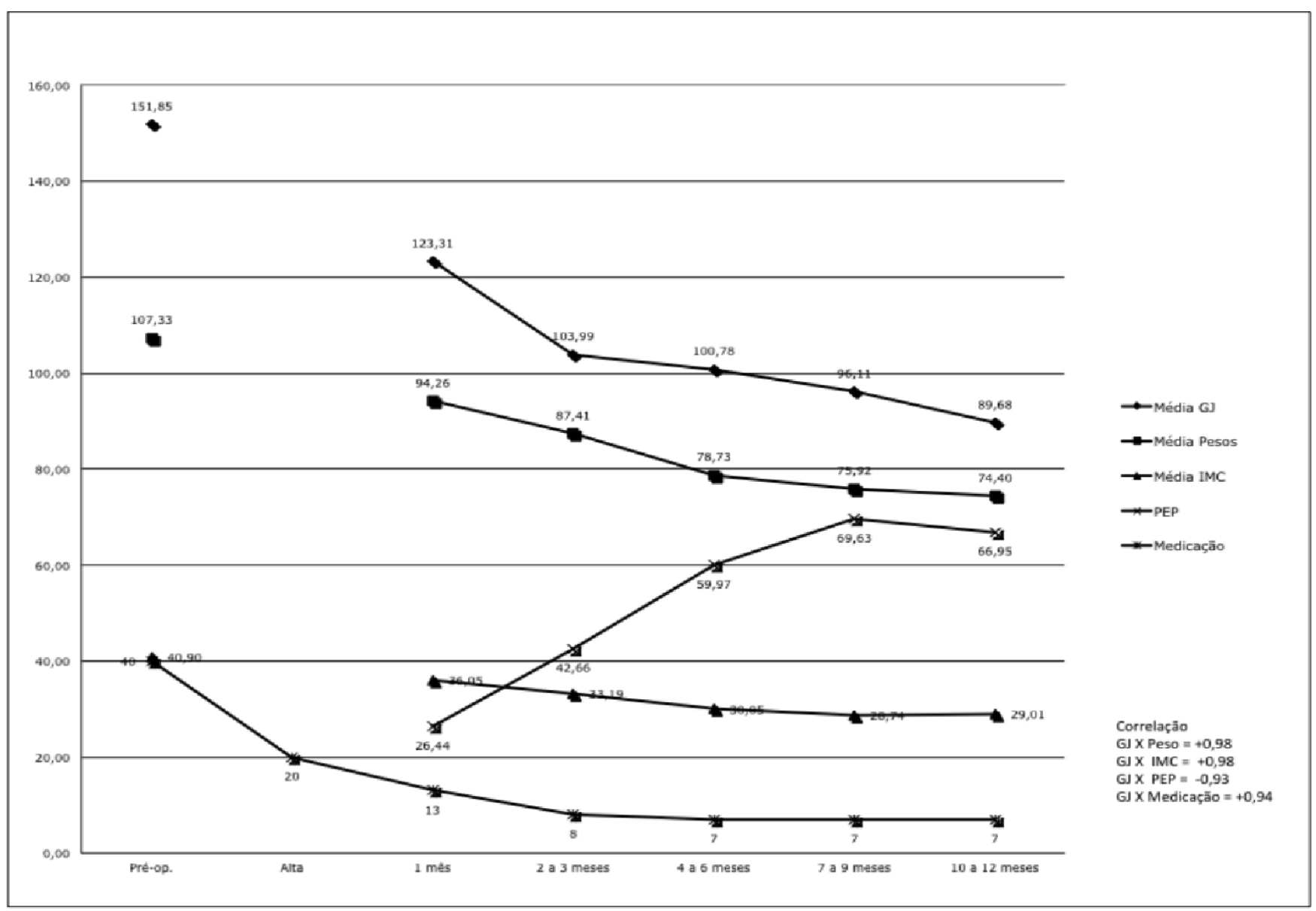

Figura 3 - Correlação entre glicemia de jejum, peso, IMC, PEP e medicação.

$G J=$ glicemia de jejum; $I M C=$ índice de massa corporal; $P E P=$ percentagem de perda de excesso de peso.

tamento cirúrgico para os pacientes obesos com DM2, o mais cedo possível| ${ }^{13,14}$.

Não existe uma definição aceita sobre o que compõe remissão do DM2 após cirurgia bariátrica ${ }^{15}$. A Associação Americana de Diabetes, em 2009, tentou estabelecer um consenso de definição de remissão para o DM2 e publicou um sumário com definições e recomendações: obtém-se, remissão parcial com valores glicêmicos entre $100-125 \mathrm{mg} / \mathrm{dl}$; remissão completa, com glicemias normais (valor $<100 \mathrm{mg} / \mathrm{dl}$ ) e um ano sem tratamento medicamentoso; e remissão prolongada quando os parâmetros se mantêm por mais de cinco anos, com hemoglobina glicada normal $(<6,5 \%)$ e sem medicação ou procedimento em andamento ${ }^{16}$. Schauer et al. num seguimento de quatro anos pós-operatório, observaram que a glicemia retornou ao valor normal em $83 \%$ e melhorou em $17 \%$ dos casos $^{12}$. Outros autores encontraram reduções significativas das glicemias compatíveis com remissão parcial do DM2, num período médio de 12 a 24 meses pós-operatório ${ }^{4,17}$. Os procedimentos de bandas gástricas ajustáveis são associados com uma média inferior (50-60\%) de remissão clínica de $\mathrm{DM} 2^{18}$.
Os estudos com bypass gástrico foram associados, em 99 a 100\% dos casos, à prevenção do diabetes em pacientes com intolerância à glicose, e a uma resolução clínica de 80 a 90\% do DM2 diagnosticado precocemente ${ }^{18}$. Num estudo realizado na França, com 35 pacientes submetidos ao bypass gástrico por laparoscopia, em que todos os pacientes eram tratados com hipoglicemiante oral ou insulina parenteral antes da operação, o DM2 foi resolvido em $60 \%$ dos casos e a redução da terapia farmacológica foi observada em 31,42\% em um ano após o procedimento ${ }^{19}$. Em vários artigos, tanto a remissão quanto a melhora do diabetes são variáveis: remissão de 58 a 89\% dos casos e melhora de 42 a 86,6\% ${ }^{20-23}$. Mottin et al. demonstraram controle do DM2, em $97,6 \%$ dos pacientes, 18 meses após bypass gástrico ${ }^{4}$. Verificamos, então, que alguns autores (quens?) utilizam o termo resolução, enquanto outros (quens?) utilizam controle e outros remissão ${ }^{24}$.

Neste estudo, a redução da glicemia aconteceu desde a alta hospitalar e, no período de sete a nove meses, as médias das glicemias de jejum apresentaram valores inferiores a $100 \mathrm{mg} / \mathrm{dl}$. A metade dos pacientes (50\%) 
suspendeu o uso de medicação para controle do DM2 na alta hospitalar e mais 13 (32,5\%) em até nove meses. Não podemos, com estes dados, avaliar a remissão, pois seria necessário um tempo maior do que um ano de acompanhamento após a operação. Entretanto, verificamos meIhora do diabetes, pois, ao final do período de 12 meses, $36(83,7 \%)$ pacientes não utilizavam mais medicação e apenas sete $(16,3 \%)$ necessitaram continuar com medicação para controle do DM2. Observamos, também, que $100 \%$ dos pacientes pararam de utilizar insulina, em até quatro meses após a operação.

A perda de peso desempenha um papel importante na remissão em longo prazo e na durabilidade da remissão ${ }^{15,25}$. A cirurgia bariátrica é, atualmente, o método mais eficiente para ocasionar perda de peso e controlar as comorbidades, sendo o bypass gástrico em $Y$ de Roux a técnica mais empregada ${ }^{26}$.

Quanto aos desfechos secundários, a perda do excesso de peso foi $66,95(+7,47 \%)$, valores superiores aos $55,9 \%$ da metanálise e revisão sistemática de Buchwald, de $2009^{23}$. Outros autores encontraram valores semelhantes $(67 \%)$ aos do nosso estudo, em um ano de acompanhamento ${ }^{20}$. Também encontramos na literatura uma redução do IMC e da perda de peso semelhantes aos deste estudo ${ }^{19,27}$

De acordo com o Ministério da Saúde, a mortalidade do diabetes está aumentando no Brasil. Os óbitos passaram de 30 para 33, por grupo de 100 mil habitantes, no período analisado (1996 a 2007). As mortes causadas pela doença cresceram em 10\%. Uma diminuição nas mortes relacionadas com o diabetes foi relatada por Laville et al. ${ }^{14}$.

A redução da mortalidade, com o tratamento cirúrgico, ocorre principalmente devido a um decréscimo no número de mortes cardiovasculares ${ }^{28}$. Um estudo observacional, mostrou que os pacientes submetidos ao bypass gástrico tiveram um decréscimo de $40 \%$ no risco relativo de morte, comparado com o grupo controle, e as mortes relacionadas ao diabetes foram reduzidas em $92 \%{ }^{29}$

A cirurgia bariátrica é uma opção de tratamento eficaz e segura para a perda de peso, aumenta a longevidade e a qualidade de vida dos obesos mórbidos e possui uma taxa aceitável de morbidade e mortalidade. A taxa de mortalidade operatória, descrita na literatura, varia de 0,1 a $2 \%$, dependendo do procedimento e das características dos pacientes ${ }^{23}$. Nossos índices de mortalidade, na cirurgia bariátrica, em publicação anterior foi $1,21 \%{ }^{30}$. Entretanto, no presente estudo não houve mortalidade.

No modelo deste estudo, após o bypass gástrico em pacientes obesos e diabéticos, observamos, no pós-operatório imediato e durante todo o período de um ano, redução das taxas glicêmicas para valores normais e redução na necessidade do uso de drogas hipoglicemiantes.

\title{
A B S T R A C T
}

\begin{abstract}
Objective: To study the effect of gastric bypass on blood glucose levels and the use of antidiabetic medication in obese patients with diabetes. Methods: We carried out a retrospective cohort study with 44 obese patients with DM2, from 469 patients undergoing gastric bypass from December 2001 to March 2009. The primary endpoints evaluated were fasting glucose and the need for antidiabetic medication. Results: The study population consisted of ten (22.7\%) men and 34 (77.3\%) women, with a mean age of $45.3( \pm 8.23)$ years and a body mass index of $40.9( \pm 5.03) \mathrm{kg} / \mathrm{m} \square$. The average time to progression of DM2 was $63.6( \pm 60.9)$ months. Of the 40 patients who used medication to control type 2 diabetes, 20 (50\%) had their medication discontinued at discharge and 13 $(32.5 \%)$, until nine months later. In one patient it was not possible to evaluate the use of medication, this being the only exception. Insulin was suspended in ten (100\%) patients who used it, six (60\%) at discharge. Fasting plasma glucose levels decreased throughout the study period $(p<0.05)$ when compared with preoperative values, and values below $100 \mathrm{mg} / \mathrm{dl}$ were achieved within seven to nine months. Conclusion: Obese patients with DM2 undergoing gastric bypass showed improved glycemic control and reduced use of hypoglycemic agents in the short-term.
\end{abstract}

Key words: Patients. Obesity. Diabetes mellitus type 2. Glycemia. Gastric Bypass.

\section{REFERÊNCIAS}

1. Amedrican Diabetes Association. Diagnosis and classification of diabetes mellitus. Diabetes Care. 2010;33(Suppl 1):S62-9.

2. Rizvi AA. Type 2 diabetes: epidemiologic trends, evolving pathogenetic [corrected] concepts, and recent changes in therapeutic approach. South Med J. 2004;97(11):107987

3. Monteiro CA, Moura EC, Conde WL, Popkin BM. Socioeconomic status and obesity in adult populations of developing countries: a review. Bull World Health Organ. 2004:82(12):940-6.
4. Mottin CC, Vontobel Padoin A, Schroer CE, Barancelli FT, Glock L, Repetto G. Behavior of type 2 diabetes mellitus in morbid obese patients submitted to gastric bypass. Obes Surg. 2008;18(2):179-81.

5. Cummings DE, Overduin J, Foster-Schubert KE, Carlson MJ. Role of the bypassed proximal intestine in the anti-diabetic effects of bariatric surgery. Surg Obes Relat Dis. 2007;3(2):109-15.

6. Mason EE. The mechanisms of surgical treatment of type 2 diabetes. Obes Surg. 2005;15(4):459-61.

7. Rubino F, Forgione A, Cummings DE, Vix M, Gnuli D, Mingrone G, et al. The mechanism of diabetes control after gastrointestinal bypass surgery reveals a role of the proximal small intestine in the pathophysiology of type 2 diabetes. Ann Surg. 2006;244(5):741-9. 
8. Ballantyne GH. Peptide YY(1-36) and peptide YY(3-36): Part II. Changes after gastrointestinal surgery and bariatric surgery. Obes Surg. 2006;16(6):795-803.

9. Pories WJ, Swanson MS, MacDonald KG, Long SB, Morris PG, Brown BM, et al. Who would have thought it? An operation proves to be the most effective therapy for adult-onset diabetes mellitus. Ann Surg. 1995;222(3):339-50; discussion 350-2.

10. Rubino F, Marescaux J. Effect of duodenal-jejunal exclusion in a non-obese animal model of type 2 diabetes: a new perspective for an old disease. Ann Surg. 2004;239(1):1-11.

11. Dixon JB. Obesity and diabetes: the impact of bariatric surgery on type-2 diabetes. World J Surg. 2009;33(10):2014-21.

12. Schauer PR, Burguera B, Ikramuddin S, Cottam D, Gourash W, Hamad $G$, et al. Effect of laparoscopic Roux-en $Y$ gastric bypass on type 2 diabetes mellitus. Ann Surg. 2003;238(4):467-84; discussion 484-5.

13. Cummings S, Apovian CM, Khaodhiar L. Obesity surgery: evidence for diabetes prevention/management. J Am Diet Assoc. 2008;108(4 Suppl 1):S40-4.

14. Laville $\mathrm{M}$, Disse E. Bariatric surgery for diabetes treatment: why should we go rapidly to surgery. Diabetes Metab. 2009;35(6 Pt 2):562-3.

15. Perugini RA, Malkani S. Remission of type 2 diabetes mellitus following bariatric surgery: review of mechanisms and presentation of the concept of 'reversibility'. Curr Opin Endocrinol Diabetes Obes. 2011;18(2):119-28.

16. Buse JB, Caprio S, Cefalu WT, Ceriello A, Del Prato S, Inzucchi SE, et al. How do we define cure of diabetes? Diabetes Care. 2009;32(11):2133-5.

17. Kim S, Richards WO. Long-term follow-up of the metabolic profiles in obese patients with type 2 diabetes mellitus after Roux-en-Y gastric bypass. Ann Surg. 2010;251(6):1049-55.

18. Ferchak CV, Meneghini LF. Obesity, bariatric surgery and type 2 diabetes - a systematic review. Diabetes Metab Res Rev. 2004;20(6):438-45.

19. Nocca D, Guillaume F, Noel P, Picot MC, Aggarwal R, El Kamel M, et al. Impact of laparoscopic sleeve gastrectomy and laparoscopic gastric bypass on $\mathrm{HbA} 1 \mathrm{c}$ blood level and pharmacological treatment of type 2 diabetes mellitus in severe or morbidly obese patients. Results of a multicenter prospective study at 1 year. Obes Surg. 2011;21(6):738-43.

20. Smith BR, Hinojosa MW, Reavis KM, Nguyen NT. Remission of diabetes after laparoscopic gastric bypass. Am Surg. 2008;74(10):948-52.
21. Alexandrides TK, Skroubis G, Kalfarentzos F. Resolution of diabetes mellitus and metabolic syndrome following Roux-en-Y gastric bypass and a variant of biliopancreatic diversion in patients with morbid obesity. Obes Surg. 2007;17(2):176-84.

22. Buchwald $H$, Avidor $Y$, Braunwald E, Jensen MD, Pories W, Fahrbach $\mathrm{K}$, et al. Bariatric surgery: a systematic review and meta-analysis. Jama. 2004;292(14):1724-37.

23. Buchwald H, Estok R, Fahrbach K, Banel D, Jensen MD, Pories WJ, et al. Weight and type 2 diabetes after bariatric surgery: systematic review and meta-analysis. Am J Med. 2009;122(3):248-56.e5.

24. Cohen R, Torres MC, Schiavon CA. Cirurgia metabólica: mudanças na anatomia gastrointestinal e a remissão do diabetes mellitus tipo 2. ABCD, arq bras cir dig. 2010;23(1):40-5.

25. Eisenberg D, Duffy AJ, Bell RL. Update on obesity surgery. World J Gastroenterol. 2006;12(20):3196-203.

26. Martins MVDC, Souza AA. Mecanismos cirúrgicos de controle do diabetes mellitus tipo 2 após cirurgia bariátrica. Rev Col Bras Cir. 2007;34(5):343-6.

27. Valezi AC, Mali Júnior J, Menezes MA, Brito EM, Souza JCL. Evolução ponderal oito anos após a derivação gástrica em Y-de-Roux. Rev Col Bras Cir. 2011;38(4):232-5.

28. MacDonald KG Jr, Long SD, Swanson MS, Brown BM, Morris $P$, Dohm GL, et al. The gastric bypass operation reduces the progression and mortality of non-insulin-dependent diabetes mellitus. J Gastrointest Surg. 1997;1(3):213-20; discussion 220

29. Adams TD, Gress RE, Smith SC, Halverson RC, Simper SC, Rosamond WD, et al. Long-term mortality after gastric bypass surgery. N Engl J Med. 2007;357(8):753-61.

30. Stoll A, Gugelmin G, Lampa Júnior VM, Frigulha C, Selbach RA. Complicações e óbitos nas operações para tratar a obesidade mórbida. ABCD, arq bras cir dig. 2011;24(4):282-4.

Recebido em 02/06/2012

Aceito para publicação em 31/07/2012

Conflito de interesse: nenhum

Fonte de financiamento: nenhuma

\section{Como citar este artigo:}

Stoll A, Silva JC, Von Bathen LC, Gugelmin G, Vedan AB, Souza BV. O efeito em curto prazo do bypass gástrico sobre pacientes obesos diabéticos. Rev Col Bras Cir. [periódico na Internet] 2013;40(1). Disponível em URL: http://www.scielo.br/rcbc

Endereço para correspondência:

Aluisio Stoll

E-mail: aluisiostoll@me.com 